\title{
PROGRAMA DE BÚSQUEDA SISTEMÁTICA DE EVIDENCIAS TEMPRANAS Y HALLAZGO DE UNA PUNTA DE PROYECTIL TIPO COLA DE PESCADO EN ALTO RÍO ÑIREHUAO
}

\author{
CÉSAR MÉNDEZ", OMAR REYES**, AMALIA NUEVO DELAUNAY"*** \& PABLO GONZÁLEZ*****
}

\begin{abstract}
RESUMEN
Se presenta el hallazgo de una punta de proyectil tipo "cola de pescado" fruto de un programa de búsqueda dirigida, diseñado para identificar geoformas de origen glacial del Pleistoceno final en el área de alto Nirehuao ( $45^{\circ} 20^{\prime}$ S, Aisén, Chile). En el marco de este programa se desarrolló una prospección identificando márgenes de un paleolago proglaciar por medio de análisis de fotos aéreas. Uno de los hallazgos más significativos fue realizado en el área de Pampa Coichel (BN 22), donde la punta de proyectil aislada fue registrada en una localidad a cielo abierto. Este resultado es discutido en función de la información arqueológica local, regional y supraregional. Finalmente, en este contexto se exploran las potencialidades de modelar paisajes antiguos aislando variables geomorfológicas que sirvan de guía para la búsqueda de evidencias tempranas.
\end{abstract}

PALABRAS CLAVE: punta de proyectil tipo "cola de pescado", Patagonia Central, transición Pleistoceno Holoceno.

\section{SYSTEMATIC SEARCH PROGRAM FOR EARLY EVIDENCE AND FINDING OF A FISHTAIL TYPE PROJECTILE POINT AT UPPER ÑIREHUAO RIVER.}

\footnotetext{
ABSTRACT

We present the finding of a "fishtail" type projectile point as result of a search program targeted to identify late Pleistocene glacial geoforms in the area of upper Nirehuao ( 45 20' S, Aisén, Chile). Framed within this program we carried out surface surveys by identifying the margins of a proglacial paleo-lake through air-photograph analysis. One of the most remarkable findings was obtained in the area of Pampa Coichel (BN 22), where the isolated lithic projectile point was recorded at an open-air location. This result

* Departamento de Antropología, Facultad de Ciencias Sociales, Universidad de Chile. C. Ignacio Carrera Pinto 1045, Nuñoa, Santiago, Chile.cmendezm@uchile.cl.

** Centro de Estudios del Hombre Austral, Instituto de la Patagonia, Universidad de Magallanes. Av. Bulnes 01890, Punta Arenas, Chile. omarreyesbaez@gmail.com.

*** Departamento de Antropología, Facultad de Ciencias Sociales, Universidad Alberto Hurtado. Almirante Barroso 10, Santiago, Chile. anuevodelaunay@gmail.com.

***** Ignacio Echeverría 8564, La Cisterna, Santiago.
} 
is discussed in the frame of local, regional and supraregional information. Finally, we explore the potential of developing a program aimed to model old landscapes by isolating geomorphological variables in order to guide the search of early evidence.

KEY WORDS: "fishtail" type projectile point, Central Patagonia, late Pleistocene.

\section{INTRODUCCIÓN}

El reconocimiento de localidades con evidencia humana de la transición Pleistoceno Holoceno en Sudamérica, y en Patagonia en particular, no ha estado generalmente relacionado a programas de búsqueda dirigida. Una proporción importante de la identificación de nuevas evidencias ha estado condicionada por los procesos formacionales que las expusieron o bien su hallazgo ocurrió a través de excavaciones donde no había forma de predecirlas (Jackson \& Méndez, 2004). En Patagonia además, la gran mayoría de las evidencias humanas fechadas hacia la transición Pleistoceno Holoceno han sido identificadas en cuevas (Borrero \& Franco, 1997; Massone, 1999; Jackson, 2007). Esta situación trae consigo como consecuencias: (1) una poca capacidad predictiva de nuevos y diferentes hallazgos y (2) el hecho que se dependa de circunstancias ocasionales para continuar aportando datos al problema (Jackson \& Méndez, 2004). En el marco de recientes investigaciones en la estepa del norte de Aisén continental, uno de los principales objetivos fue poner a prueba un programa de búsqueda que permitiera evaluar deliberadamente el hallazgo de evidencia temprana y sus condiciones. Para ello se reflexionó sobre cuáles serían las expectativas para hallar evidencias materiales de la transición Pleistoceno Holoceno y se desarrolló un programa ad-hoc a estos propósitos.

En esta breve comunicación presentamos los resultados de las actividades de prospección realizadas en el área de la cuenca alta del río Nirehuao. Este sector extra andino corresponde a un área de extensos planos depositacionales bordeados por lomajes suaves reconocidos genéricamente como Relieves Planiformes Orientales (SERPLAC, 2005). Destaca en el paisaje el Complejo Volcánico Baño Nuevo de tobas de lapilli y brechas piroclásticas que se hace visible en la forma de promontorios destacados en un amplio sector rellenado por depósitos glaciales de fondo (Suárez et al., 2007). Al estar localizado al este de los Andes, las precipitaciones del Cinturón de Vientos del Oeste se ven condicionadas por el efecto orográfico (Garreaud, 2009). Consecuentemente, el piso vegetacional del sector corresponde a la estepa mediterránea-templada de Festuca pallescens y Mulinum spinosum y limita hacia el oeste con un bosque caducifolio templado andino de Nothofagus pumilio y Berberis ilicifolia (Luebert \& Pliscoff, 2006).

\section{EL PROGRAMA DE BÚSQUEDA Y LA METODOLOGÍA DE PROSPECCIÓN}

Las evidencias recuperadas en el sitio El Chueco 1 (44²9'37” S) (Reyes et al., 2007; Méndez et al., 2011) y Baño Nuevo 1 (45¹7'36" S) (Mena \& Stafford 2006, Reyes et al., 2012), indicaban que dentro de la región de Aisén (Fig. 1), las zonas esteparias orientales eran las áreas más propicias para la búsqueda de evidencia humana de la transición Pleistoceno/Holoceno. Particularmente en El Chueco 1, una excavación profunda $(230 \mathrm{~cm})$-en condiciones donde no se presumía ningún hallazgo temprano- permitió registrar evidencias con una edad de $10.100 \pm 40$ años ${ }^{14} \mathrm{C}$ AP $(11.760-11.270$ años cal AP; Reyes et al., 2007); respondiendo a un modo de hallazgo fortuito. Trabajos posteriores en el área inmediata (Alto Cisnes) permitieron no sólo posicionar estas evidencias en el marco una reconstrucción paleoambiental que se extendía a todo el Holoceno (De Porras et al., 2012), sino también delimitar un extenso paleolago de origen glaciar que hipotéticamente habría condicionado la disponibilidad de espacios durante el Pleistoceno final (Méndez et al., 2009). Estos antecedentes llevaron a seleccionar dos nuevas áreas: Alto Simpson ( 45 $55^{\circ}$ S) y Alto Nirehuao $\left(\sim 45^{\circ} 15^{\prime}\right.$ S), como potenciales sectores para replicar estos hallazgos, aunque en esta ocasión, mediados por una búsqueda dirigida. Ambos sectores se caracterizan por ser áreas de estepa abierta, con geoformas de origen glaciar y oferta de reparos rocosos. Los resultados obtenidos 


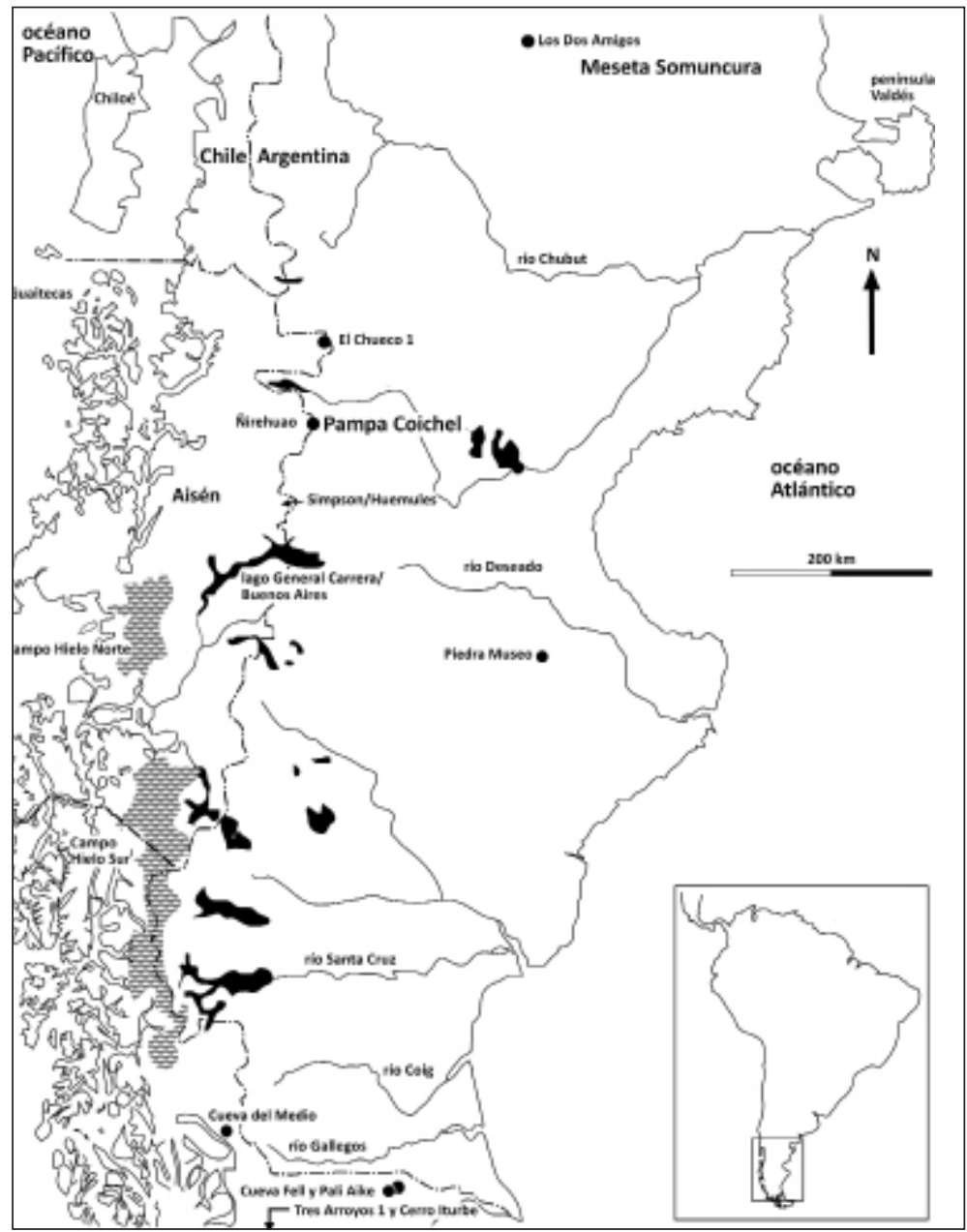

Fig. 1. Mapa de Patagonia con las localidades mencionadas en el texto.

en el área de Alto Simpson fueron negativos para la identificación de información de la transición Pleistoceno/Holoceno, sin embargo, resultaron muy útiles para a afinar criterios metodológicos relativos a la búsqueda mediada por la reconstrucción dirigida por geoformas (Méndez et al., 2012).

En el área de Alto Ñirehuao sí se obtuvieron resultados positivos. La evidencia previa constaba de un conjunto de evidencias arqueológicas y bioarqueológicas en la cueva de Baño Nuevo 1 fechadas a partir de los $9.530 \pm 25$ años ${ }^{14} \mathrm{C} \mathrm{AP} \mathrm{(11.070} \mathrm{a}$ 10.700 años cal AP) (Mena \& Stafford, 2006). Éstas, sin embargo, post datan las evidencias paleontológicas que se inician hacia los $13.480 \pm 35$ años ${ }^{14} \mathrm{C}$ AP (16.430 a 15.950 años cal AP) en un depósito que marca el fin de la incorporación de sedimentos arenosos al interior de la cueva (Mena \& Stafford, 2006). Las cartas geológicas disponibles corroboran la existencia de depósitos de origen glaciolacustre extendidos en parte del curso alto del valle de Nirehuao (Suárez et al., 2007). Con el fin de entender la evolución geomorfológica de esta área se realizó un análisis estereoscópico de 23 fotos aéreas, foto interpretación y un mapeo de escala 1:70.000 (Ortega, 2011). Las fotos aéreas fueron digitalizadas, georeferenciadas y rectificadas mediante los programas ArcGIS y ENVI 4.0. (Datum WGS 84, Zona 19) y se incorporó la información aportada por la imagen satelital Landsat L7123091_09120051212 y los datos de elevación del Shuttle Radar Topography Mission (s46w072) (Ortega, 2011).

A partir de este trabajo se discriminó una 


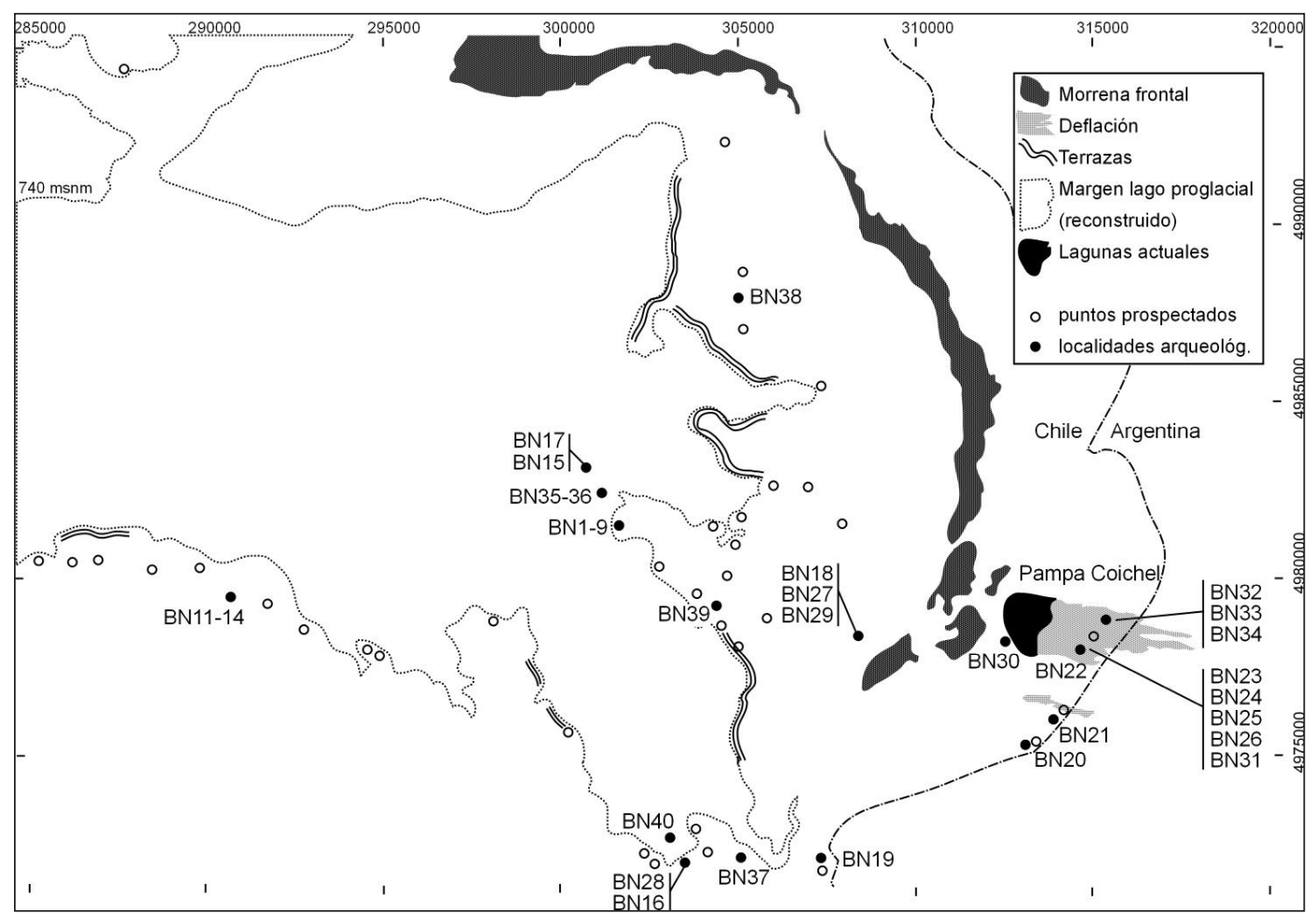

Fig. 2. Mapa del área de estudio, cuenca del Alto Nirehuao, de acuerdo a una reconstrucción paleoambiental, puntos prospectados (solo 32) y sitios discutidos en el texto, (BN: Baño Nuevo).

serie de geoformas de origen glaciar, entre las que se incluían morrenas, abanicos aluviales de apariencia deltaica, paleocanales de drenaje y cuatro niveles de paleocostas de origen lacustre, de las cuales dos (740 y 770 msnm, respectivamente), eran más marcadas y recurrentes a lo largo del valle (Ortega, 2011). Estas geoformas, fuera de ratificar la fuerte impronta modeladora de los glaciares en el área de estudio, apuntaban a la existencia de un paleolago proglaciar contemporáneo o anterior a los fechados más tempranos (>16.500 años cal AP) obtenidos en el sitio Baño Nuevo 1.

Este estudio permitió definir una serie de puntos que guiaron la búsqueda arqueológica. Dada la mayor representación espacial de las paleocostas de los 740 y 770 msnm, se optó por usarlas como guías para la prospección pedestre dirigida. La disposición del sitio de Baño Nuevo 1 por sobre la cota de $740 \mathrm{msnm}$, aunque no brinda certeza del momento exacto en que el paleolago estuvo a ese nivel, sí proponía un límite tentativo de las áreas a recorrer. Otro límite lo proveyó la exclusión de los sectores que pudieran haber estado obliterados o tapados por remociones en masa. Adicionalmente, se dispusieron puntos de prospección en áreas más alejadas de las cotas a fin de recorrer espacios que pudieran haber estado siempre disponibles para la ocupación humana (Fig. 2).

Se definieron un total de 46 puntos para guiar la prospección: 15 a nivel de la cota de 770 msnm correspondiente a la paleocosta más antigua, 17 a nivel de la cota de 740 msnm, y 14 fuera de la influencia directa de los paleolagos. De los últimos, se localizaron: siete puntos al norte, tres al sur en el área de Punta del Monte y cuatro al este en el área de Pampa Alta/Coichel. Otro límite para establecer la distribución de los puntos de prospección fue la divisoria limítrofe entre Chile y Argentina. Para la definición de los puntos se privilegió tanto áreas con disponibilidad de reparos rocosos, como sectores a cielo abierto. En torno a cada punto se realizó un recorrido pedestre en un radio de $0,5 \mathrm{~km}$, donde se describieron las geoformas principales y los hallazgos materiales. No se efectuaron sondeos.

Los resultados de esta prospección fueron limitados en cuanto a información distribucional ya 


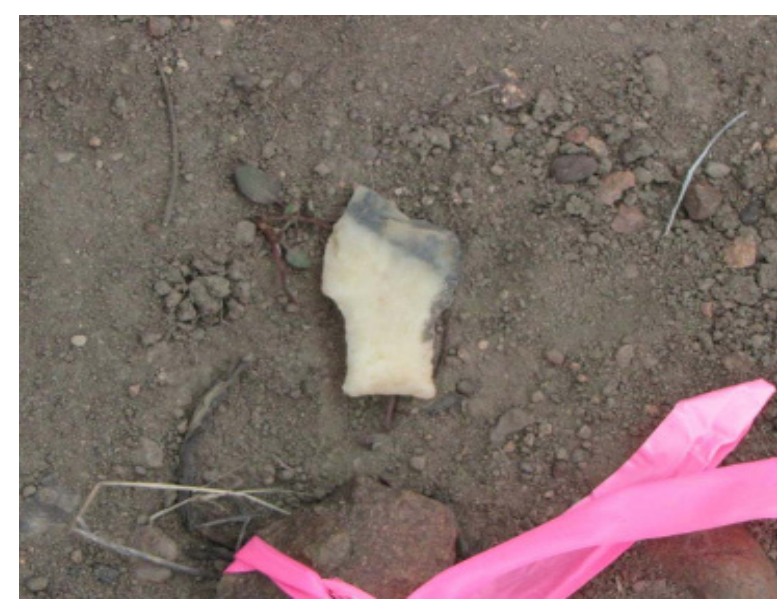

Fig. 3. Imagen in situ de punta de proyectil cola de pescado de Pampa Coichel (BN 22).

que no pretendieron recorridos en amplias áreas. Esto produjo que también fueran bajas las frecuencias materiales. A la fecha se registran 40 localidades de interés arqueológico en el área (BN01 a BN40). Éstas incluyen 17 sitios arqueológicos (sean reparos rocosos con ocupación humana o dispersiones superficiales de material lítico), 15 hallazgos aislados y ocho reparos rocosos con potencial aún no determinado. De estos puntos, nueve se conocían antes de nuestros trabajos en la zona (BN01 a BN08,

García, 2006; Mena comunicación personal 2013) y 31 se registraron recientemente. Sin embargo, de los últimos, sólo 19 corresponden a sitios $(\mathrm{N}=10)$ o hallazgos aislados $(\mathrm{N}=9)$ que fueron identificados en asociación a los puntos predefinidos que guiaron la prospección (dentro o cercanos al radio de recorrido). Todos estos corresponden a localidades a cielo abierto con variables -aunque bajas- frecuencias de material lítico en superficie. De los puntos registrados destacó el hallazgo de una punta de proyectil tipo "cola de pescado" en las inmediaciones de Pampa Coichel.

\section{CONTEXTO DE HALLAZGO Y DESCRIPCIÓN DE LAS PIEZAS}

Pampa Coichel corresponde a una vasta área a 850 msnm en promedio, donde un campo de dunas activas de $4 \mathrm{~km}^{2}$ de arenas, limos y arcillas bien seleccionadas (Suárez et al., 2007), se dispone entre laguna Coichel (fuente de sedimentos) y la frontera entre Chile y Argentina $(<2 \mathrm{~km})$. Corresponde a

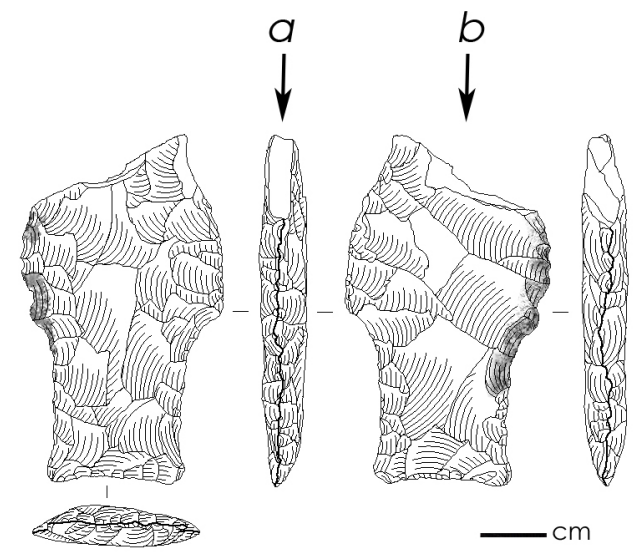

Fig. 4. Dibujo técnico punta de proyectil tipo cola de pescado de Pampa Coichel (BN 22), a: golpe de buril, b. fractura en bisagra, oscurecido: presencia de líquenes.

una zona de alta actividad eólica con amplias áreas de deflación que permite la exposición ocasional de pequeños perfiles estratigráficos que exhiben suelos antiguos. En los sectores erosionados se expone material arqueológico con distintos grados de erosión diferencial en sus caras. En este contexto se registró una punta de proyectil tipo "cola de pescado" (BN22, datum WGS84; 45¹9'47.1'S, 71²1'36.39”'W, 851 msnm) de sílice blanca con una banda diagonal color negra, con el ápice fracturado (Fig. 3 y 4).

Se desconoce la procedencia de la materia prima por cuanto no se dispone de caracterizaciones regionales de los recursos líticos. Las medidas de la pieza fueron tomadas siguiendo los parámetros sugeridos por Morrow \& Morrow (1999). Éstas son: (a) ancho máximo 25,6 mm; (b) altura del ancho máximo: 29,8 mm; (c) ancho basal: $17 \mathrm{~mm}$; (d) profundidad de la concavidad basal: 0,8 $\mathrm{mm}$; (e) espesor máximo: 5,65 mm.

En términos técnicos, se observan cicatrices de adelgazamiento que se disponen principalmente de forma diagonal al eje central de la pieza. En ambas caras, las lascas de adelgazamiento superan el eje medio de la pieza y no se registra acanaladura en ninguna de ellas. Se observa retoque subparalelo a lo largo de todo el contorno de la pieza. Ambas secciones, tranversal y longitudinal, son biconvexas. No son evidentes signos de desgaste intencional en los bordes del pedúnculo, aunque sí en la concavidad basal. Todas estas observaciones sugieren que la pieza se encontraba terminada y en un estadio aprovechable para su funcionamiento. La pieza 


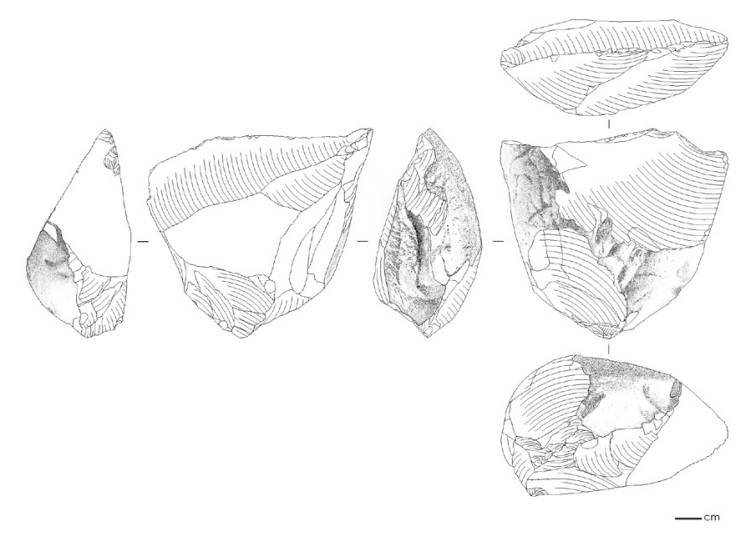

Fig. 5. Dibujo técnico instrumento informal de Pampa Coichel (BN 22).

muestra dos fracturas en la parte distal, referidas en la figura por las letras a y b. La primera se dispone a lo largo de un margen de la pieza y se asemeja a un golpe de tipo "buril" que se dispone adyacente a un lascado con la misma dirección. Por su parte, la segunda es una fractura en bisagra más grande y corresponde a la mayor parte de la remoción del segmento distal de la pieza. Los atributos de percusión sugieren que ambas fracturas se originaron en el extremo distal de la pieza, por lo que se infiere un origen común, probablemente generado por un impacto contra un sustrato duro. Estos dos tipos de fracturas son esperables en actividades de caza como lo sugieren estudios experimentales replicativos (Titmus \& Woods, 1986; Flenniken, 1990) y estudios específicos sobre este tipo de puntas (Weitzel, 2012). No hay ninguna indicación de reactivado o retomado (cambio de función) de la pieza posterior a su fractura. Finalmente, la cara que se identificó expuesta hacia arriba mostró una ligera mayor erosión que la cara en contacto con la superficie y se observaron líquenes en el sector mesial de uno de sus márgenes y por ambas caras (sector reparado de los vientos dominantes).

A escasos 2 metros de la pieza se registró un núcleo/tajador con huellas de uso (Fig. 5). Esta pieza posee escasos lascados por talla intencional y huellas macroscópicas de uso, aunque la fuerte erosión sobre sus caras ha borrado la definición de algunas aristas. No existen otras indicaciones que permitan sugerir contemporaneidad entre ambas piezas.

\section{COMENTARIOS}

Aunque la mayoría de las puntas de proyectil tipo "cola de pescado" en Sudamérica proceden de contextos sin cronología conocida, aquellas asociadas a depósitos fechados se localizan en la transición Pleistoceno-Holoceno (Politis, 1991; Flegenheimer et al., 2003). En Patagonia, este tipo de puntas de proyectil han sido registradas en las localidades de Cueva de Fell, Pali Aike (Bird, 1993), Cueva del Medio (Nami, 1985-6), Tres Arroyos 1 (Jackson, 2002; Massone, 2004), Piedra Museo (Miotti, 1995), Los Dos Amigos Oeste (Miotti et al., 2010), y de forma aislada en Estancia Pali Aike (Nami, 1994), Cerro Iturbe (Jackson, 2004) y en colecciones (Bahamondes \& Jackson, 2006). Si bien la cronología asociada a este tipo de cabezales en Patagonia requiere aún precisarse, un recuento de los fechados más confiables permite sugerir su ocurrencia en el intervalo entre 11.100 a 10.400 años ${ }^{14} \mathrm{C}$ AP $(\sim 12.980$ a 12.270 años cal AP) (Nami \& Nakamura, 1995; Prates et al., 2013), sin que esto permita descartar eventuales evidencias fuera de este rango. Por ello es posible considerarlas como un apropiado marcador temporal para construir hipótesis respecto a los espacios disponibles para los primeros pobladores de la región (Miotti et al., 2010, 2012).

En la región de Aisén sólo se contaba con información previa de dos piezas bifaciales de basalto de grano fino descritas por Bate (1982) y Sade (2007), respectivamente, como preformas de este tipo de cabezal, ambas procedentes de la zona de las cabeceras del río Simpson/Huemules. Dado que las piezas provienen de colecciones particulares y su locación de hallazgo es desconocida, resultan poco útiles para inferir con precisión la disponibilidad de espacios para el uso humano durante la transición Pleistoceno Holoceno. Por el contrario, el hallazgo del cabezal lítico de Pampa Coichel permite precisar espacios efectivamente ocupados y cercanos a las márgenes de los glaciares en momentos de profundos cambios vegetacionales, hidrológicos y geomorfológicos en la región (Villa-Martínez et al., 2011; De Porras et al., 2012). No existen fechados para el vaciamiento del paleolago y sin dudas, éste podría corresponder a un evento anterior al arribo de los humanos a la región. Adicionalmente, no puede desestimarse el rol de las condiciones de visibilidad en áreas de deflación como Pampa Coichel. Sin 
embargo, los resultados revisten importancia para continuar las exploraciones ya que, siguiendo un protocolo metodológico con base en las geoformas, se logró recuperar evidencia singular, que se asocia al bloque temporal estudiado.

La comparación entre fragmentación de las puntas de proyectil y su lugar de descarte es informativa del uso diferencial de los espacios (Knecht, 1997). Particularmente en estas piezas, los daños ocurridos por impacto nos revelan información de su contexto de uso, ya que se asume que en la localidad de descarte, los usuarios eventualmente estuvieron en capacidad de reemplazar por un cabezal nuevo (Andrefsky, 2010). Resulta lógico suponer que este tipo de actividades ocurrieran insertas en las actividades de caza o alternativamente que la punta de proyectil aislada podría corresponder a un cabezal perdido, ya que los artefactos que se mueven más durante su uso tienen mayores probabilidades de pérdida (Schiffer, 1996).

Distintos equipos de investigación han decidido abordar la búsqueda de evidencias tempranas a través de la reconstrucción de los ambientes potenciales y condicionantes que facultarían su presencia $\mathrm{y} / \mathrm{o}$ su hallazgo (e.g. Jackson et al., 2007; McCulloch \& Morello, 2009; Martin \& San Román, 2010; Santoro et al., 2011). La implementación de este tipo de metodología demuestra que, reconociendo aspectos formacionales, geográficos y ambientales, es viable orientar las búsquedas hacia determinados bloques temporales. Esta elección no es excluyente de otras metodologías que puedan igualmente guiar la búsqueda de registro arqueológico. En esta ocasión se optó por aislar variables geomorfológicas y modelar algunos de los aspectos más notorios de los paisajes antiguos. Dichos parámetros fueron implementados en la discriminación de áreas para recorrer, potencialmente utilizables por sociedades pasadas, y permitieron el hallazgo de una pieza, cuyas cualidades formales son sugerentes de una cronología relativa a la transición PleistocenoHoloceno. Aunque aún no poseemos edades ${ }^{14} \mathrm{C}$ para precisar la cronología de las evidencias de Pampa Coichel y reconocemos que éste es sólo un hallazgo aislado, proponemos que lo más relevante es reconocer el potencial del uso de la geomorfología en la definición de expectativas de hallazgo. Nuestra orientación no sólo probó ser útil para establecer las localidades con hallazgos, sino también aportó a definir ejemplos de los espacios ocupados para actividades a cielo abierto.

\section{AGRADECIMIENTOS}

Financiado por FONDECYT 1130128. Agradecemos a Estancia Baño Nuevo y a los Srs. Claudio Bariggi, Robinson Palma, Freddy Boldt y Eloy Rivera por las facilidades y ayuda en nuestra investigación. Agradecemos a Valentina Trejo, Francisco Mena, Héctor Velásquez, Catalina Contreras, Juan García, Antonio Maldonado, Rafael Goñi, Kurt Rademaker, María Luisa Gómez, Ricardo Labra por su participación en esta prospección, a Donald Jackson su ayuda en el análisis de la punta de proyectil, a Paulina Chávez por los dibujos técnicos y a Nora Franco por oportunos comentarios a una versión anterior del manuscrito.

\section{BIBLIOGRAFÍA}

Andrefsky, W. (2010). Human land use strategies and projectile point damage, resharpening and discard patterns. Human Evolution, 25(1-2), 13-30.

Bahamondes, F. \& Jackson, D. (2006). Hallazgo de una punta "cola de pescado" en Magallanes, Chile. Magallania, 34(2), 115-118.

Bate, L. (1982). Los origenes de la comunidad primitiva en Patagonia. Ciudad de México: Editorial Universitaria.

Bird, J. (1993). Viajes y Arqueología en Chile Austral. Punta Arenas: Ediciones de la Universidad de Magallanes.

Borrero, L. \& Franco, N. (1997). Early Patagonian hunter-gatherers: subsistence and technology. Journal of Anthropological Research, 53, 219-239.

De Porras, Maldonado, M. A., Abarzua, A., Cárdenas, M., Francois, J. P., Martel-Cea, A., Stern, C, Méndez, C \& Reyes, O. (2012). Postglacial vegetation, fire and climate dynamics at Central Patagonia (Lake Shaman, $44^{\circ}$ S), Chile.Quaternary Science Reviews, 50, 71-85.

Flegenheimer, N., Bayón, C., Valente, M., Baeza J., \& Femenías, J. (2003). Long distance tool stone transport in the Argentine Pampas. Quaternary International, 109-110, 49-64.

Flenniken, J. (1990). Stone tool reduction techniques as cultural markers. En M. Soto de Arechavaleta (Ed.), Nuevos enfoques en el estudio de la lítica (pp.189-204). México DF: Universidad Autónoma de México.

García, C. (2006). Los materiales líticos de las ocupaciones 
de cazadores recolectores en la cueva Baño Nuevo -1. En F. Mena y V. Lucero (Comps.), Informe proyecto FONDECYT 1030560 año 3. Santiago: CONICYT.

Garreaud R. (2009). The Andes climate and weather. Advances in Geosciences, 22, 3-11.

Jackson, D. (2002). Los instrumentos líticos de los primeros cazadores de Tierra del Fuego. Ensayos y Estudios. Santiago: DIBAM.

Jackson, D. (2004). Hallazgo de una punta "Cola de Pescado" en Patagonia Meridional. Magallania, 32, 221-223.

Jackson, D. (2007). Estructura, intensidad y reiteración en las ocupaciones paleoindias en cuevas y aleros de Patagonia Meridional (Chile). Cazadores Recolectores del Cono Sur, 2, 67-87.

Jackson, D. \& Méndez C. (2004). Hallazgo o búsqueda de sitios paleoindios: problemas de investigación en torno a los primeros poblamientos. Werken, 5, 9-14.

Jackson, D., Méndez, C., Seguel, R., Maldonado, A. \& Vargas, G. (2007). Initial occupation of the Pacific coast of Chile during Late Pleistocene times.Current Anthropology, 48(5), 725-731.

Knecht, H. (1997). The history and development of projectile technology research. En H. Knecht (Ed.), Projectile technology (pp. 3-35). New York: Plenum Press.

Luebert, F. \& Pliscoff, P. (2006). Sinopsisbioclimática y vegetacional de Chile. Santiago: Editorial Universitaria.

Massone, M. (1999). Aproximación metodológica al estudio de las ocupaciones tempranas de los cazadores terrestres en la Región de Magallanes. En J.B. Belardi, P.M. Fernández, R.A. Goñi, A.G. Guráieb\& M. De Nigris (Eds.), Soplando en el Viento. Actas de las Terceras Jornadas de Arqueología de la Patagonia (pp. 99-112). Neuquén y Buenos Aires: Instituto Nacional de Antropología y Pensamiento Latinoamericano y Universidad Nacional de Comahue.

Massone, M. (2004). Los cazadores después del hielo. Centro de Investigaciones Barros Arana. Santiago: DIBAM.

Martin F., \& San Román, M. (2010). Explorando la variabilidad del registro arqueológico y tafonómico en Pali-Aike (Chile) a través de la búsqueda de registros Pleistocenos a cielo abierto. Magallania, 38(1), 199-215.

McCulloch, R. \& Morello, F. (2009). Evidencia glacial y paleoecológica de ambientes tardiglaciales y del Holoceno temprano. Implicaciones para el poblamiento temprano de Tierra del Fuego. En F. Salemme, F. Santiago, M. Álvarez, E. Piana, M. Vázquez \& E. Mansur (Eds.), Arqueología de Patagonia: una mirada desde el último confin, (pp. 119-133). Ushuaia: Editorial Utopías.

Mena, F. \& Stafford, T. (2006). Contexto estratigráfico y fechación directa de esqueletos humanos del Holoceno Temprano en Cueva Baño Nuevo 1 (Patagonia Central, Chile). En J. Jiménez, S. González, J. Pompa \& F. Ortíz (Eds.), Segundo Simposio Internacional del Hombre Temprano en América, (pp. 139-54). Ciudad de México: INAH.

Méndez, C., Reyes, O., Maldonado, A., \& François, J.P. (2009). Ser humano y medio ambiente durante la transición Pleistoceno Holoceno en las cabeceras del río Cisnes ( 44ํㅇ, Aisén Norte). En M. Salemme, F. Santiago, M. Álvarez, E. Piana, M. Vázquez \& E. Mansur (Eds.), Arqueología de Patagonia: una mirada desde el último confín, (pp. 75-83). Ushuaia: Editorial Utopías.

Méndez, C., Reyes, O., Nuevo Delaunay, A., Trejo, V., Barberena R., \& Velásquez, H. (2011). Ocupaciones humanas en la margen occidental de Patagonia Central: eventos de poblamiento en Alto Río Cisnes. Magallania, 39, 223-242.

Méndez, C., Reyes, O., Trejo V., \& Nuevo Delaunay, A. (2012). Ocupación humana de alto río Simpson, Aisén (margen occidental de la estepa de Patagonia Central) como caso para medir la intensidad de uso de espacios. En A.F. Zangrando, R. Barberena, A. Gil, G. Neme, M. Giardina, L. Luna, C. Otaola, S. Paulides, L. Salgán\& A. Tivoli (Eds.), Tendencias teórico-metodológicas y casos de estudio en la arqueología de la Patagonia (pp. 193-201). Buenos Aires: Museo de Historia Natural de San Rafael-Sociedad Argentina de AntropologíaInstituto Nacional de Antropología y Pensamiento Latinoamericano.

Miotti, L. (1995). Piedra Museo locality: a special place in the New World. Current Research in the Pleistocene, 12, 37-40.

Miotti, L., Hermo D., \& Terranova, E. (2010). Fishtail points, first evidence of Late-Pleistocene hunter-gatherers in Somuncurá plateau (Río Negro Province, Argentina). Current Research in the Pleistocene, 27, 22-24.

Miotti, L., Terranova, E., Barberena, R., Hermo, D., Giesso, M., \&Glascock, M. (2012). Geochemical sourcing of obsidian fishtail points: studies for the Somuncurá Plateau (Río Negro, Argentina). En L. Miotti, M. Salemme, N. Flegenheimer \& T. Goebel(Eds.), Southbound: Late Pleistocene peopling of Latin America, (pp. 127-131). Oregon: Center for the Study of the First Americans, College Station.

Morrow, J. \& Morrow, T. (1999). Geographic variation in fluted projectile points: a hemispheric perspective. American Antiquity, 64(2), 215-231.

Nami, H. (1985-6). Excavación arqueológica y hallazgo de una punta de proyectil "Fell I" en la Cueva del Medio, seno de Última Esperanza, Chile. Informe Preliminar. Anales del Instituto de la Patagonia. Serie Ciencias 
Humanas, 16,103-109.

Nami, H. (1994). Reseña sobre los avances de la arqueología finipleistocénica del extremo sur de Sudamérica. Chungara, 26(2), 145-163.

Nami, H. \&Nakamura, T. (1995). Cronología radiocarbónica con AMS sobre muestras de hueso procedentes del sitio Cueva del Medio. Anales del Instituto de la Patagonia. Serie Ciencias Humanas, 23,125-133.

Ortega, C. (2011). Geomorfología del área Ñireguao - Baño Nuevo - Coyhaique Alto. En C. Méndez y O. Reyes (Comps.), Informe proyecto FONDECYT 1090027 año 3. Santiago: CONICYT.

Politis, G. (1991). Fishtail projectile points in southern cone of South America: an overview. En R. Bonnichsen\& K. Turnmire (Eds.), Clovis: origins and adaptations, (pp. 287-301). Oregon: Center for the Study of the First Americans.

Prates, L., Politis, G. \& Steele, J. (2013).Radiocarbon chronology of the early human occupation of Argentina.Quaternary International 301, 104-122.

Reyes, O., Méndez, C., Mena, F. \& Moraga, M. (2012).The bioanthropological evidence of a ca. 10,000 CALYBP ten-individual group from Central Patagonia. En L. Miotti, M. Salemme, N. Flegenheimer y T. Goebel (Eds.), Southbound: Late Pleistocene peopling of Latin America (pp. 39-43). Oregon: Center for the Study of the First Americans, College Station.

Reyes, O., Méndez, C., Trejo, V., \& Velásquez, H. (2007). El Chueco 1: un asentamiento multicomponente en la estepa occidental de Patagonia Central (11400 a 2700 años cal. AP., $44^{\circ}$ S). Magallania, 35(1),61-74.

Sade, K. (2007). Cazadores extintos de Aysén continental: propuesta de poblamiento. Coihaique: Ediciones Nire Negro.

Santoro, C., Ugalde, P., Latorre, C., Salas, C., Osorio, D., Jackson, D., \& Gayó, E. (2011). Ocupación humana Pleistocénica en el Desierto de Atacama. Primeros resultados de la aplicación de un modelo predictivo de investigación interdisciplinaria. Chungara Revista de Antropología Chilena, 43 (número especial), 357-366.

Serplac. (2005). Atlas de la Región de Aysén. Santiago: Ministerio de Planificación y Cooperación, LOM Ediciones. Schiffer, M. (1996).Formation processes of the archaeological record. University of Utah Press, Salt Lake City.

Suárez, M., De La Cruz, R. \& Bell, M. (2007). Geología del área Nireguao-Baño Nuevo. Región Aisén del General Carlos Ibáñez del Campo. Carta Geológica de Chile. SerieGeologíaBásica. Santiago: SERNAGEOMIN.

Titmus, G. \& Woods J. (1986). An experimental study of projectile point fracture patterns. Journal of California and Great Basin Anthropology, 8(1), 37-49.

Villa-Martínez, R., Moreno, P. \& Valenzuela, M. (2011). Deglacial and postglacial vegetation changes on the eastern slopes of the central Patagonian Andes (47 $\left.{ }^{\circ} \mathrm{S}\right)$. Quaternary Science Reviews, 32, 86-99.

Weitzel, C. (2012). Broken stone tools from Cerro El Sombrero Cima (Tandilia Range, Argentina). En L. Miotti, M. Salemme, N. Flegenheimer \& T. Goebel, (Eds.), Southbound: Late Pleistocene peopling of Latin America, (pp 111-115). Oregon: Center for the Study of the First Americans, College Station. 
